Article

\title{
Electro-Thermal Formation of Uniform Lying Helix Alignment in a Cholesteric Liquid Crystal Cell
}

\author{
Chia-Hua Yu, Po-Chang Wu and Wei Lee *(i) \\ Institute of Imaging and Biomedical Photonics, College of Photonics, National Chiao Tung University, Guiren \\ Dist., Tainan 71150, Taiwan; rickyu@ms69.hinet.net (C.-H.Y.); jackywu@nctu.edu.tw (P.-C.W.) \\ * Correspondence: wlee@nctu.edu.tw; Tel.: +886-6-303-2121 (ext. 57826); Fax: +886-6-303-2535
}

Received: 1 March 2019; Accepted: 26 March 2019; Published: 1 April 2019

\begin{abstract}
We demonstrated previously that the temperature of a sandwich-type liquid crystal cell with unignorable electrode resistivity could be electrically increased as a result of dielectric heating. In this study, we take advantage of such an electro-thermal effect and report on a unique electric-field approach to the formation of uniform lying helix (ULH) texture in a cholesteric liquid crystal (CLC) cell. The technique entails a hybrid voltage pulse at frequencies $f_{1}$ and, subsequently, $f_{2}$, which are higher and lower than the onset frequency for the induction of dielectric heating, respectively. When the cell is electrically sustained in the isotropic phase by the voltage pulse of $V=35 \mathrm{~V}_{\mathrm{rms}}$ at $f_{1}=55 \mathrm{kHz}$ or in the homeotropic state with the enhanced ionic effect at $V=30 \mathrm{~V}_{\mathrm{rms}}$ and $f_{1}=55 \mathrm{kHz}$, our results indicate that switching of the voltage frequency from $f_{1}$ to $f_{2}$ enables the succeeding formation of well-aligned ULH during either the isotropic-to-CLC phase transition at $f_{2}=1 \mathrm{kHz}$ or by the electrohydrodynamic effect at $f_{2}=30 \mathrm{~Hz}$. For practical use, the aligning technique proposed for the first time in this study is more applicable than existing alternatives in that the obtained ULH is adoptable to CLCs with positive dielectric anisotropy in a simple cell geometry where complicated surface pretreatment is not required. Moreover, it is electrically switchable to other CLC textures such as Grandjean planar and focal conic states without the need of a temperature controller for the phase transition, the use of ion-rich LC materials, or mechanical shearing for textural transition.
\end{abstract}

Keywords: dielectric heating; cholesteric liquid crystals; uniform lying helix

\section{Introduction}

Cholesteric liquid crystals (CLCs) constitute a class of soft photonic crystal, with a self-assembled, helically molecular configuration in one-dimension. Considered in a confined geometry, three main CLC states, namely, Grandjean planar (P), focal conic (FC), and fingerprint (FP), distinctly in molecular orientation and optical properties, have been well distinguished. The molecular helix (helical axis) of a CLC cell in the P state is oriented perpendicular to the substrate normal and it can reflect circularly polarized light with the same handedness as that of the CLC, following Bragg's reflection law. The FC state is found to have randomly oriented molecular helices so that it can scatter unpolarized light due to the mismatch of refractive indices between domain walls. For the FP state where the molecular helix lies in the plane of substrates, unpolarized light passing through it could be either diffracted or transmitted depending on the relation between the wavelength of light and the length of helical pitch [1]. Accordingly, by virtue of unique optical properties and textural switching, CLCs have long been potential candidates for applications in photonics as switchable and memorable optical devices [2-4]. On the other hand, when the helical pitch length is comparable to or shorter than visible light wavelengths, the CLC can also be regarded as a uniaxial birefringent medium analogously to achiral nematic liquid crystals (LCs) with the helical axis being the optical axis. The uniform lying helix (ULH) is a class of this kind of short-pitch CLCs in the FP state with the helical helix oriented in the 
substrate plane along a preferred direction. In contrast to the aforementioned CLC textures, the ULH is attractive for its superior electro-optic responses, enabling the modulations in phase retardation and intensity of polarized light under crossed polarizers by the processes of helix-unwinding and in-plane switching of optic axis via the voltage-induced dielectric and flexoelectric effects, respectively [5]. Particularly, the flexoelectro-optic switching of ULH structures with a wide viewing angle and short response time $(<1 \mathrm{~ms})$ have been proven by extensive studies, including those using bimesogenic LCs [6-8] and others concerning doping bent-core dimers into rod-like CLCs [9-11]. Owing to the luring uniqueness, the ULH structure has received a lot of interest, extending the application domain of CLCs to next-generation displays, such as ultra-high resolution TVs, virtual reality and augmented reality head-mounted displays, glasses-free 3D displays, and field-sequential color displays, whose images need to be displayed with high definition and high refresh rate.

The most severe problem impeding practical uses of the ULH is the difficulty in obtaining a defect-free alignment in a simple cell geometry. This is not surprising because a CLC in the cell with either planar or homeotropic alignment surfaces is favorably stabilized in the P or FC state to minimize the free energy. For this purpose, technologies based on electric-field and surface-alignment pretreatments to induce the ULH alignment have successively been exploited with a varying degree of limitations. The earliest and the most commonly adopted electric-field method was reported by Lee and Patel, in which a temperature-cooling process is essential for the CLC cell to go through the isotropic-to-CLC phase transition when an AC voltage is applied [12]. The optic axis of the obtained ULH is formed with a deviation angle from the rubbing direction. On the basis of this method, Salter et al. reported that the resulting ULH in a planar-aligned cell with $90^{\circ}$-twisted rubbing is more uniform than those of hybrid-aligned cells and of the planar-aligned cells with parallel $\left(0^{\circ}\right.$ or $180^{\circ}$ ) rubbing [13]. Without undergoing the temperature-cooling process, voltage-generated ULH alignment has also been obtained in planar-aligned CLC cells by providing an extra mechanical force to induce shear flow in the meantime [14-16], using ion-rich positive nematic host to permit electrohydrodynamic flow by low-frequency voltages [17,18], controlling voltage conditions precisely to induce the nematic-to-CLC structural transition [19], optimizing the pretilt angle as well as the anchoring energy [20], or by designing a tri-electrode configuration [21,22]. For the surface-treatment method, the concept stems from the modification in surface morphology of alignment layers, enabling periodic anchoring for realizing the ULH structure spontaneously. This prerequisite has been implemented by using cholesteric alignment layers with the same pitch as that of injected material [23], coating weak homeotropic alignment films on scratched substrates [24], generating interdigital planar and homeotropic channels on substrates [25], and creating grooves by means of two-photon excitation laser lithography [26], mold-templating [27], and by laser writing [28]. In some cases of the above, the isotropic-to-CLC temperature cooling process with and without the assistance of the electric field was required to ensure the uniformity of ULH. However, the manufacturing processes of ULH by surface-treatment methods would become too complicated, especially for mass production.

Recently, we have demonstrated that the dielectric heating effect can be induced in a non-dual-frequency CLC cell by high-frequency voltages (e.g., negative chiral nematic in [29]). As confirmed experimentally and theoretically, this is caused by the pseudo-dielectric relaxation from the non-ideal cell geometry with finite conductivity in indium-tin-oxide (ITO) electrodes rather than the intrinsic molecular rotation. Based on this electro-thermal effect, we propose, in this work, to form ULH structures by applying a hybrid pulse with a fixed amplitude and frequencies $f_{1}$ and $f_{2}$ $\left(<f_{1}\right)$ across a CLC cell thickness. The pulse at $f_{1}$ is responsible for elevating the cell temperature. Switching the frequency from $f_{1}$ to $f_{2}$ enables the formation of ULH alignment during the phase transition or via the electrohydrodynamic instability. In the following, we first clarify the mechanism of the voltage-induced textural and phase transition of the CLC by dielectric heating. The processes of forming ULH alignment by the treatment of designated hybrid pulse are then manifested. 


\section{Experimental}

The CLC used in this study was a mixture composed of $70 \mathrm{wt} \%$ of a positive nematic LC (E44, Daily Polymer Co., Kaohsiung, Taiwan) and $30 \mathrm{wt} \%$ of the left-handedness chiral additive S811 $(\mathrm{HCCH})$. Material properties of E44 give the refractive indices $n_{\mid l}=1.79$ and $n_{\perp}=1.53$ (measured at the wavelength of $589 \mathrm{~nm}$ and temperature of $20^{\circ} \mathrm{C}$ ), the twist elastic constant $K_{22}$ of $13 \mathrm{pN}$, and dielectric anisotropy $\Delta \varepsilon=14.38$ as determined by the difference between the parallel and perpendicular components of dielectric constant of $\varepsilon_{||}=19.42$ and $\varepsilon_{\perp}=5.04$, respectively (at the frequency of $1 \mathrm{kHz}$ and temperature of $20^{\circ} \mathrm{C}$ ). The CLC was put on a hot stage, heated to the isotropic phase, stirred for two hours, and finally injected by capillary action into an empty cell with a planar surface alignment and $90^{\circ}$-twisted rubbing [19]. Cell parameters, including the electrode area $A=10 \times 10 \mathrm{~mm}^{2}$, cell gap $d=4.8 \pm 0.5 \mu \mathrm{m}$, and the ITO electrode resistivity $R_{\text {ITO }} \sim 310 \Omega$, were identical to those reported in [29] so that the dielectric heating effect could be expectedly induced by optimized voltage conditions. The clearing point $T_{\mathrm{c}}$ (i.e., the transition temperature between the isotropic and CLC phases) of the CLC cell, as determined by temperature-dependent dielectric spectroscopy together with textural observations, was $57.0^{\circ} \mathrm{C}$ and the CLC phase could be preserved at least down to $0{ }^{\circ} \mathrm{C}$. Dielectric spectra of the CLC cell at designated temperatures were acquired with an LCR meter (Agilent E4980A) and a temperature controller (Linkam T95-PE). The adjustable amplitude and frequency ranges of the sinusoidal probe voltage provided by the LCR meter were $0.05 \mathrm{~V}-20 \mathrm{~V}$ and $20 \mathrm{~Hz}-2 \mathrm{MHz}$, respectively. The CLC cell was driven by square-wave voltage supplied from an arbitrary function generator (Tektronix AFG-3022B) in conjunction with an amplifier (TREK Model 603). The types of CLC textures and their uniformity were preliminarily examined by textural observation using a polarizing optical microscope (Olympus BX51) in the transmission mode. The optical transparency or voltage-dependent transmission of CLC textures was quantitatively inspected by setting the cell between a He-Ne laser source with an emission wavelength of $632.8 \mathrm{~nm}$ and a photodetector without any polarizer. A non-contact IR thermometer (IR camera FLIR ThermaCam ${ }^{\circledR}$ P25) was employed to monitor the variation in cell temperature as a function of the applied voltage.

\section{Results and Discussion}

\subsection{Frequency-Modulated Textural and Phase Transitions}

Figure 1a shows the voltage-dependent transmission $(V-T \%)$ curves of the CLC cell at a fixed temperature of $T=25^{\circ} \mathrm{C}$ as measured without any polarizer. Here, the temperature was precisely controlled by situating the cell in a temperature controller. In the case of the frequency at $f=1 \mathrm{kHz}$, the resulting $V-T \%$ curve illustrates the change of CLC texture from the initial P state to the FC state and finally to the homeotropic $(\mathrm{H})$ state with increasing voltage. This follows the general case for the voltage-induced textural transition of a CLC cell with positive dielectric anisotropy according to the dielectric coupling between LC molecules and electric fields. Accordingly, the $V-T \%$ curves at given frequencies in Figure 1a can be divided into five voltage regions, separated by colors, corresponding to specific CLC states and textural transitions. Because the wavelength of the light source (i.e., $\mathrm{He}-\mathrm{Ne}$ laser) at $632.8 \mathrm{~nm}$ is outside the (Bragg) reflection bandgap of the CLC, the transmittance $(\sim 80 \%)$ of the cell in P state $\left(\sim 80 \%\right.$ in region $\left.1, V<6 \mathrm{~V}_{\text {rms }}\right)$ is comparable to that of the voltage-sustained $\mathrm{H}$ state $\left(\sim 82 \%\right.$ in region $\left.5, V>38 \mathrm{~V}_{\text {rms }}\right)$. The voltage range (region $3,6 \mathrm{~V}_{\text {rms }}<V<38 \mathrm{~V}_{\text {rms }}$ ) resulting in a much-lowered transmittance $(T \%<8 \%)$ corresponds to the cell's FC state that effectively scatters visible light (at the wavelength of $632.8 \mathrm{~nm}$ ) due to its randomly oriented and broken helices in the bulk. The voltage region 2 (4), showing decreased (increased) transmittance from $80 \%(5 \%)$ to $5 \%$ $(82 \%)$ with ascending voltage between $6 \mathrm{~V}_{\mathrm{rms}}\left(28 \mathrm{~V}_{\mathrm{rms}}\right)$ and $28 \mathrm{~V}_{\mathrm{rms}}\left(38 \mathrm{~V}_{\mathrm{rms}}\right)$, is thus attributable to the P-to-FC (FC-to-H) textural transition. Besides, in a special case where the frequency of the applied voltage is low enough to bring about electrohydrodynamic instability (EHDI), the ULH state can be obtained in a given voltage region. This phenomenon has been evidenced in some of the early works $[17,18,30]$. However, when the frequency varies from $1 \mathrm{kHz}$ to $30 \mathrm{~Hz}$ in this study, the $V-T \%$ 
curve remains nearly unchanged as shown in Figure 1a. This implies that the ionic effect in our used CLC cell is weak and the frequency of the voltage at $30 \mathrm{~Hz}$ is still insufficient to onset the EHDI; thus, the optical signal corresponding to the ULH state is absent in Figure 1a.
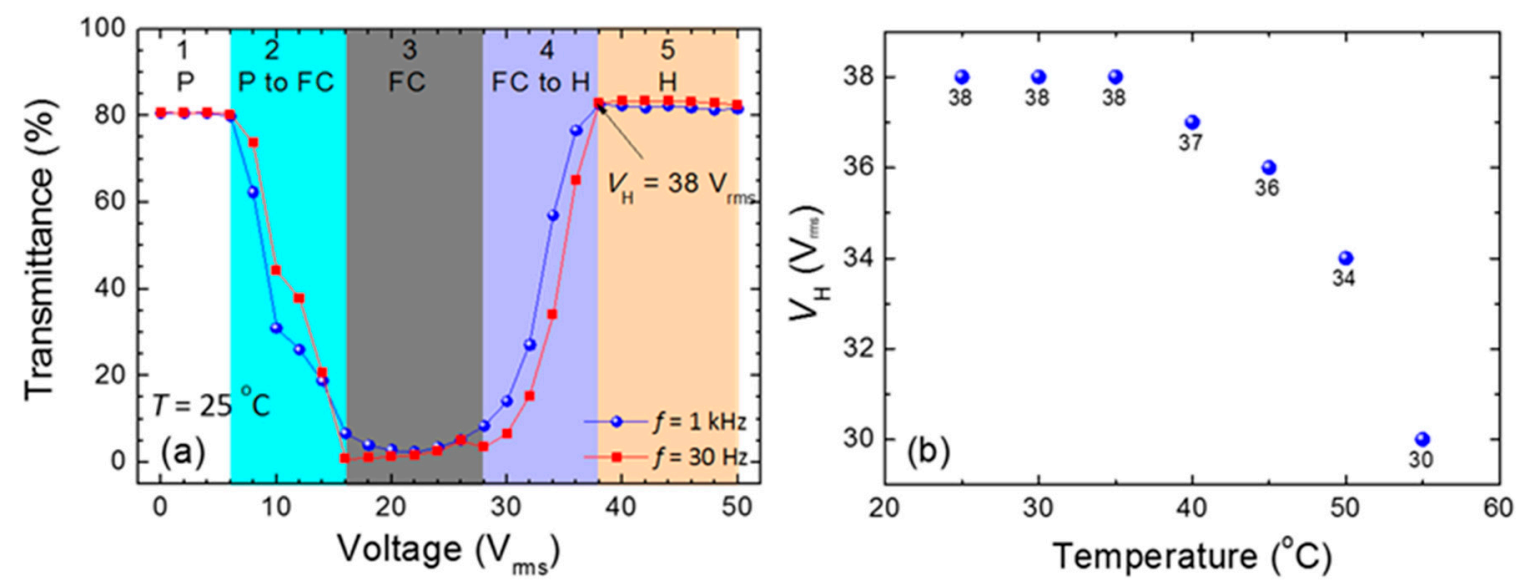

Figure 1. (a) Voltage dependence of the transmission of the CLC cell under AC voltage applied at $T=25^{\circ} \mathrm{C}$ and $(\mathbf{b})$ the change of the critical voltage $\left(V_{\mathrm{H}}\right)$ for helix unwinding as a function of temperature. Regions 1, 3, and 5 in Figure 1a are voltage ranges for sustaining the cell in the P, FC, and $\mathrm{H}$ states, respectively. Regions 2 and 4 reveal $V-T \%$ behaviors, dominated by the $\mathrm{P}$ to $\mathrm{FC}$ and $\mathrm{FC}$ to $\mathrm{H}$ textural transitions, respectively.

The voltage $V_{\mathrm{H}}$ required for unwinding the CLC helix as well as sustaining the cell in the $\mathrm{H}$ state can be expressed as

$$
V_{\mathrm{H}}=\frac{\pi^{2} d}{P} \sqrt{\frac{K_{22}}{\varepsilon_{0} \Delta \varepsilon}}
$$

where $P$ stands for the helical pitch length and $\varepsilon_{0}\left(=8.854 \times 10^{-12} \mathrm{~F} \cdot \mathrm{m}^{-1}\right)$ represents the permittivity in a vacuum. One can see from Figure $1 \mathrm{~b}$ that the magnitude of $V_{\mathrm{H}}$ decreases from $38 \mathrm{~V}_{\mathrm{rms}}$ to $30 \mathrm{~V}_{\mathrm{rms}}$ as the temperature increases from $25{ }^{\circ} \mathrm{C}$ to $55^{\circ} \mathrm{C}$. In most of thermotropic calamitic or rod-like LCs, the orientational order parameter $(S)$ decreases with increasing temperature. Since the twist elastic constant $K_{22}$ and the dielectric anisotropy $\Delta \varepsilon$ are proportional to $S^{2}$ and $S$, respectively, the change in $V_{\mathrm{H}}$ as a function of temperature is primarily attributable to the substantial variation in temperature-dependent $K_{22}$.

Furthermore, Figure 2 depicts the applied (voltage) frequency dependence of the steady-state cell temperature and transmission (Figure 2a) as well as optical textures of the CLC cell subjected to a $35-\mathrm{V}_{\mathrm{rms}}$ voltage at three distinct frequencies (Figure $2 \mathrm{~b}-\mathrm{d}$ ). Here the measurements were performed without using a temperature controller. The cell temperature in the field-off state was ca. $24.4^{\circ} \mathrm{C}$, namely, the room temperature. Each cell-temperature data point was acquired by holding the given voltage applied to the cell for $10 \mathrm{~min}$ to ensure that the cell temperature reached the steady state and became time-independent. It was tested preliminarily that the onset frequency $f_{\text {th }}$ for the induction of dielectric heating by the applied voltage at $V=35 \mathrm{~V}_{\mathrm{rms}}$ is approximately $10 \mathrm{kHz}$. As revealed in Figure $2 \mathrm{a}$, the temperature of the cell grows with ascending frequency but this trend becomes mitigated as the frequency is higher than $55 \mathrm{kHz}$. This is in good agreement with our previous work in which the temperature increase, arising from significant dielectric heating from the pseudo-dielectric relaxation, is a function of the applied frequency [29,31]. In the case of $f=20 \mathrm{kHz}$, the temperature is elevated by 3.6 ${ }^{\circ} \mathrm{C}$ (i.e., $T=28,0^{\circ} \mathrm{C}$ at $f=20 \mathrm{kHz}$ ); thus, the texture (Figure 2b) under applied voltage $V=35 \mathrm{~V}_{\text {rms }}$ (lower than $V_{\mathrm{H}}=38 \mathrm{~V}_{\mathrm{rms}}$ at $T=30.0^{\circ} \mathrm{C}$ ), corresponding to region 4 in Figure 1a, is preserved in an intermediate state between $\mathrm{FC}$ and $\mathrm{H}$ state with low transmission of $\sim 27.5 \%$. As the frequency is promoted from $20 \mathrm{kHz}$ to $45 \mathrm{kHz}$, the temperature increases monotonically from $28.0^{\circ} \mathrm{C}$ to $46.4{ }^{\circ} \mathrm{C}$. In the meantime, 
the driving voltage (i.e., $V=35 \mathrm{~V}_{\text {rms }}$ ) approaches $V_{\mathrm{H}}=36 \mathrm{~V}_{\text {rms }}$ at $T=45^{\circ} \mathrm{C}$. In consequence, the sharp increase in transmittance to $\sim 78 \%$ with increasing frequency in the neighborhood of $45 \mathrm{kHz}$, as shown in Figure 2a, is undoubtedly contributed by the process of the FC-to-H phase transition (Figure 2c). Once the frequency goes beyond $55 \mathrm{kHz}$ such that the temperature showing $T=58.0^{\circ} \mathrm{C}$ becomes higher than the clearing temperature $\left(T_{\mathrm{C}}=57.0^{\circ} \mathrm{C}\right)$, the phase transition from the CLC phase to the isotropic phase will be induced. Although the optical textures under crossed polarizers and transmittances without a polarizer in both the isotropic phase and the $\mathrm{H}$ state are comparable or virtually identical, the voltage-induced isotropic phase can be confirmed by comparing the optical textures between the electrode and non-electrode areas. As evidenced in Figure 2d, the texture of the cell in the non-electrode regions around the electrode area gradually changes from the CLC to the isotropic phase owing to the diffusion of heat generated from the electrode. This observation suggests that the textural and phase transitions in the CLC cell can alternatively be induced by modulating the frequency of the applied voltage to control the cell temperature via dielectric heating [29]. From the results above, one can form a well-aligned and switchable ULH texture by treating a CLC cell with a specific AC voltage.

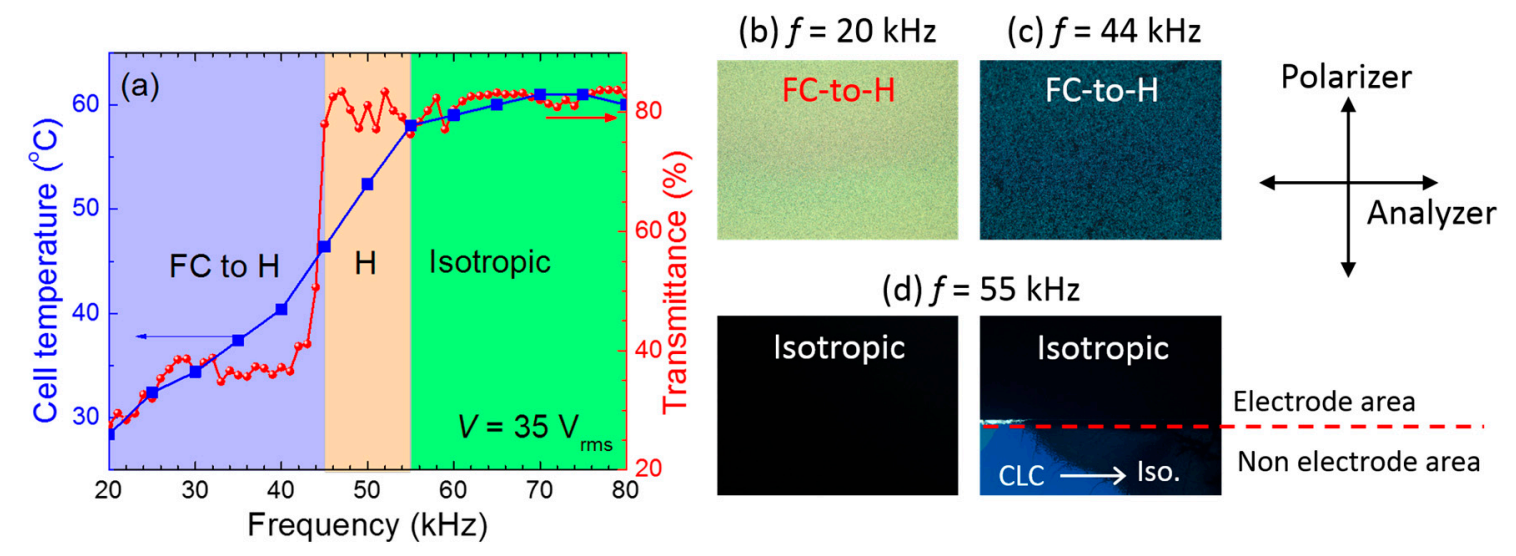

Figure 2. (a) Frequency-dependent cell temperature and transmission of the CLC cell driven by a square-voltage at $V=35 \mathrm{~V}_{\mathrm{rms}}$ and the corresponding optical textures at (b) $f=20 \mathrm{kHz},(\mathbf{c}) f=44 \mathrm{kHz}$, and $(\mathbf{d}) f=55 \mathrm{kHz}$. For $(\mathbf{b}-\mathbf{d})$, the transmission axes of the analyzer $(\mathrm{A})$ and the polarizer $(\mathrm{P})$ are crossed to each other.

Figure 3 delineates the idea of the proposed electric-field approach to the formation of well-aligned $\mathrm{ULH}$ and the scheme for the textural switching. Here, an applied voltage signal, consisting of two pulse components with one at $f_{1}$ followed instantly by the other at a lowered frequency $f_{2}$, is designed for carrying out the switching of the cell from either the P or FC state to the ULH state. The magnitudes of $f_{1}$ and $f_{2}$ designated satisfy the conditions of $f_{1}>f_{\text {th }}$ and $f_{2}<f_{\text {th }}$, where $f_{\text {th }}$ is the onset frequency for the induction of the dielectric heating in the cell. Such a pulse functions like a heat generator at $f_{1}$, enabling the temperature increase of the cell to a given value; it also serves as an agitator at $f_{2}$ to provoke phase and textural transitions. As such, there are two ways, alternative to ideas reported in $[12,17]$, to form the ULH alignment by optimizing voltage conditions of the hybrid pulse. Referring to Figure 2a, the first way is to first sustain the cell in the isotropic phase (at an arbitrary temperature beyond the clearing point) by setting the amplitude and frequency of the leading portion of the pulse to be $V=35 \mathrm{~V}_{\mathrm{rms}}$ and $f_{1}=55 \mathrm{kHz}$. When the pulse frequency is switched from $f_{1}$ to $f_{2}$ whereas the voltage magnitude remains unchanged, the dielectric heating behavior disappears, and the ULH results at $f_{2}$ during the isotropic-to-CLC phase transition. In comparison with the method reported in [12], using the designated hybrid pulse to form ULH structure would be more feasible because an additional heating stage is not required to monitor the temperature variation for the phase transition. Our method further permits the ULH to be electrically switchable by following the general scheme for switching CLC textures. As illustrated in Figure 3, the obtained ULH can be readily switched back to the P state 
by triggering the cell with a single pulse of $V=V_{\mathrm{H}}$ at $f=f_{2}$, and to the FC state by a hybrid pulse at $f_{2}$ with amplitudes of $V_{\mathrm{H}}$ for the leading component and $V<V_{\mathrm{H}}$ for the succeeding component. The second way is to generate ULH via EHDI by elevating the temperature to promote the ionic effect rather than requiring the LC material to be ion-rich $[17,18]$. In this case, the cell is held in the $\mathrm{H}$ state at a temperature close to $T_{\mathrm{c}}$ by the first portion of the pulse at $V=30 \mathrm{~V}_{\mathrm{rms}}$ and $f_{1}=55 \mathrm{kHz}$. It has been demonstrated that the ionic effect in an LC cell is enhanced at a higher temperature thanks to the reduction in molecular ordering and increase in the activation energy of mobile ions [32]. Therefore, it is expected to obtain ULH by optimizing $f_{2}$ to meet the frequency responsible for inducing the EHDI.

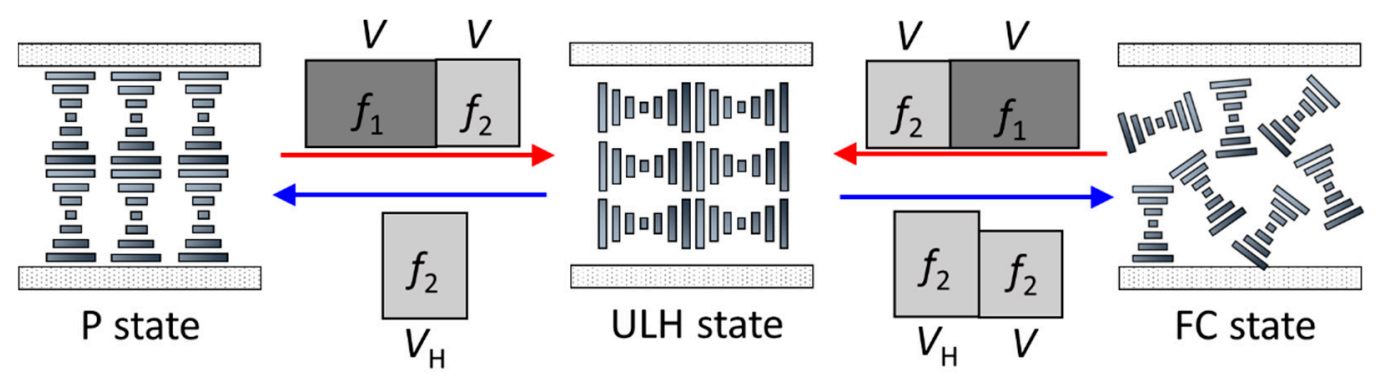

Figure 3. Schematic of the generation of the ULH state and the scheme for textural switching after voltage treatment. Note that $f_{1}>f_{2}$.

\subsection{Formation of ULH Alignment after the Treatment of a Designated Hybrid Pulse}

Following the switching scheme illustrated in Figure 3, Figure 4 shows the processes of the generation of ULH alignment in the CLC cell driven by a hybrid voltage. The total duration of the applied pulse is $810 \mathrm{~s}$. In the case of the ULH formation through the isotropic-to-CLC phase transition, $V=35 \mathrm{~V}_{\mathrm{rms}}$ and $f_{1}=55 \mathrm{kHz}$ are fixed. As shown in Figure $4 \mathrm{a}$, the cell is brought to the isotropic phase by the pulse at $f_{1}$ for $600 \mathrm{~s}$. Once the pulse is switched from $f_{1}$ to $f_{2}=30 \mathrm{~Hz}$ or $1 \mathrm{kHz}$, the isotropic-to-CLC phase transition occurs within $30 \mathrm{~s}$. Meanwhile, the ULH starts to appear and is completely formed throughout the cell at time $t=810 \mathrm{~s}$. The optic axis of this ULH alignment is deviated by an angle of $\sim 45^{\circ}$ from the rubbing direction because of the use of a $90^{\circ}$-twisted planar-aligned cell $[13,19]$. As a result, by rotating the optic axis of ULH parallel to the transmission axis of either of crossed polarizers, excellent dark appearance can be secured, indicating the uniform ULH alignment. Note that the ULH generated is a metastable state which can be retained for several hours at room temperature after voltage removal. The stability of the ULH can further be promoted by optimizing the cell-gap-to-pitch ratio [17] or by performing polymer stabilization in addition. When $V$ is reduced from $35 \mathrm{~V}_{\mathrm{rms}}$ to $30 \mathrm{~V}_{\mathrm{rms}}$, the temperature of the cell driven by the pulse at $f_{1}$ for $600 \mathrm{~s}$, as determined by a thermograph camera, is about $55.2{ }^{\circ} \mathrm{C}$, which is lower than $T_{\mathrm{C}}=57.0^{\circ} \mathrm{C}$. The dark state of the cell under crossed polarizers thus corresponds to the $\mathrm{H}$ state. Because $V=30 \mathrm{~V}_{\mathrm{rms}}$ is very close to $V_{\mathrm{H}}=30 \mathrm{~V}_{\mathrm{rms}}$ at $T=55{ }^{\circ} \mathrm{C}$ (Figure $1 \mathrm{~b}$ ), the textural transition is induced quickly after switching the pulse frequency to $f_{2}=30 \mathrm{~Hz}$ for $10 \mathrm{~s}$ (i.e., $t=610 \mathrm{~s}$ in Figure $4 \mathrm{~b}$ ), allowing the ULH to be obtained within the next $20 \mathrm{~s}$. However, if $f_{2}$ is changed from $30 \mathrm{~Hz}$ to $1 \mathrm{kHz}$, the FC state instead of the ULH state is generated (Figure $4 \mathrm{~b}$ ). This implies that the ULH formed by the $30 \mathrm{~Hz}$ voltage pulse might be a result of the electrohydrodynamic effect (to be discussed later).

The results mentioned above are further substantiated by measuring the time-dependent transmission curves of the CLC cell within the time of the voltage pulse at $f_{2}$. As shown in Figure $5 \mathrm{a}$, except for the case where the cell exhibits relatively low transmittance $(\sim 26 \%)$ due to the formation of FC state by $V=30 \mathrm{~V}_{\text {rms }}$ at $f_{2}=1 \mathrm{kHz}$, the transmittances of the other three textures with ULH alignment in the entire measured time are higher than $70 \%$, which are only about $10 \%$ lower than that of the $\mathrm{H}$ state. The wavy variation in specific temporal regions in these three curves might be attributable to the phase or the textural transition and to the variation in refractive indices with temperature over time. Figure $5 \mathrm{~b}$ displays the time-dependent temperature and temperature increment of the cell subjected to 
30- and $35-\mathrm{V}_{\mathrm{rms}}$ hybrid pulses. Driving the cell at $f_{1}=55 \mathrm{kHz}$ causes the cell temperature to increase, reaching saturation at $T=58.0^{\circ} \mathrm{C}$ for $V=35 V_{\mathrm{rms}}$ and $T=55.2^{\circ} \mathrm{C}$ for $V=30 \mathrm{~V}_{\mathrm{rms}}$ at $t \sim 200 \mathrm{~s}$; thus, holding the cell in the isotropic phase and the $\mathrm{H}$ state in the CLC phase, respectively. At the time when the pulse is changed from $55 \mathrm{kHz}$ to $f_{2}=30 \mathrm{~Hz}$, the temperature drops with ascending time and returns to the initial value (i.e., $T=24.4^{\circ} \mathrm{C}$ ) within $300 \mathrm{~s}(t \sim 900 \mathrm{~s})$. The result revealed in Figure $5 \mathrm{~b}$ also indicates that the isotropic-to-CLC phase transition at $V=35 \mathrm{~V}_{\text {rms }}$ and the H-to-CLC textural transition can be induced within $30 \mathrm{~s}$ after the frequency is switched from $f_{1}$ to $f_{2}$.

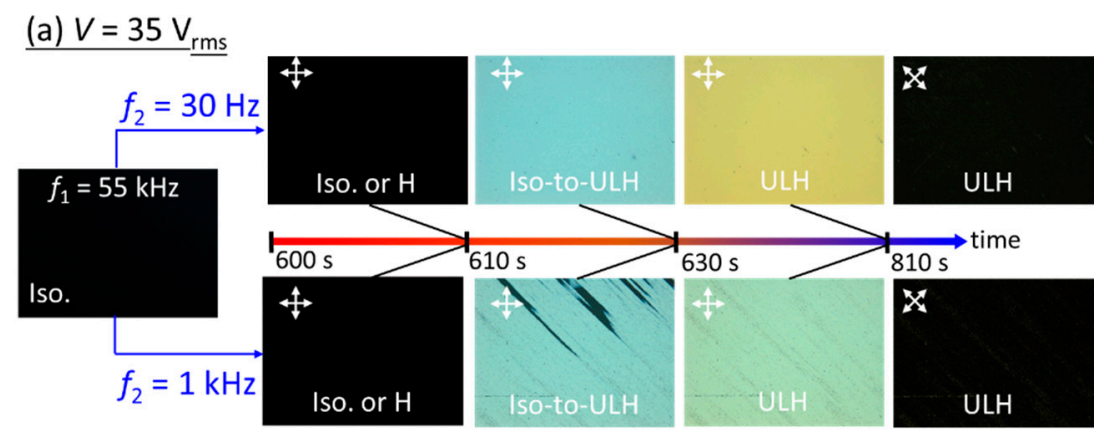

(b) $V=30 V_{r m s}$

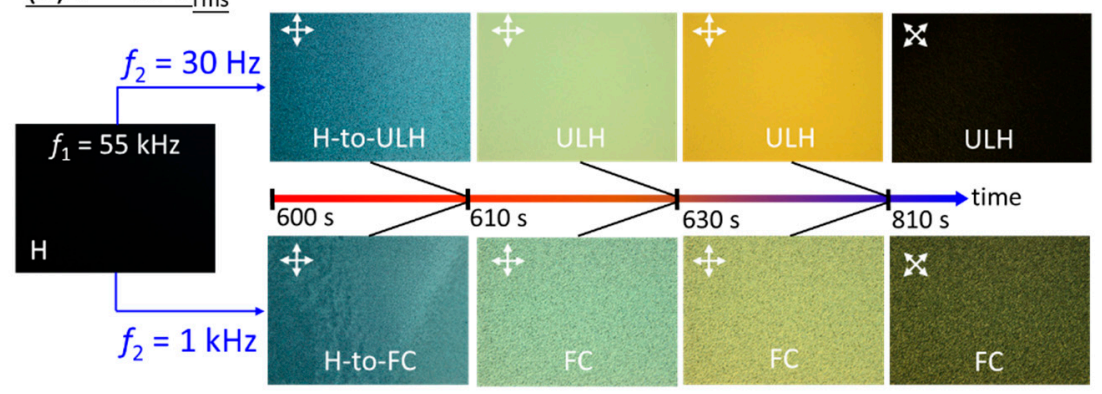

Figure 4. Processes of the formation of ULH alignment in the CLC cell during the application of separate hybrid pulses of (a) $V=35 \mathrm{~V}_{\mathrm{rms}}$ and (b) $V=30 \mathrm{~V}_{\mathrm{rms}}$ at $f_{1}=55 \mathrm{kHz}$ and $f_{2}=30 \mathrm{~Hz}$ or $1 \mathrm{kHz}$. The duration at $f_{1}$ lasts for $600 \mathrm{~s}$.
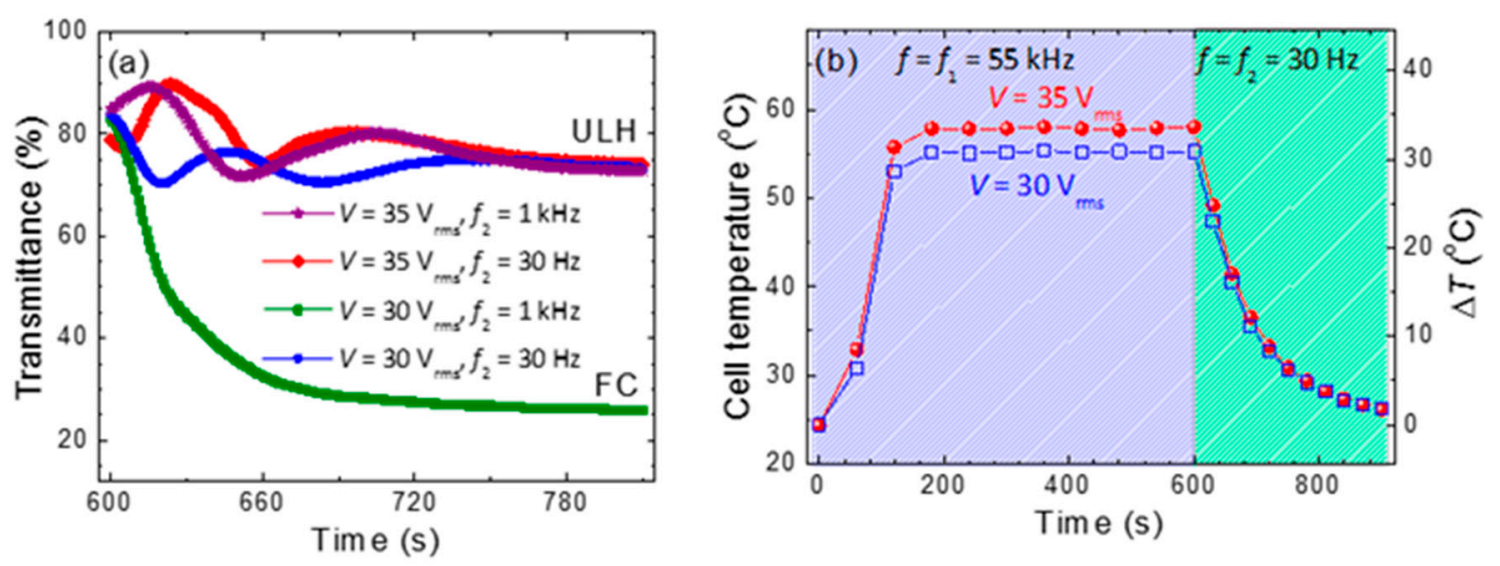

Figure 5. (a) Time-dependent transmission curves of the cell after the switching of the frequency of the hybrid pulse from $f_{1}(=55 \mathrm{kHz})$ to $f_{2}$ and (b) time-dependent temperature and temperature variation of the cell during the application of hybrid pulses at $V=35 \mathrm{~V}_{\mathrm{rms}}$ and $V=30 \mathrm{Vrms}$. The duration of the pulse at $f_{1}$ is $600 \mathrm{~s}$.

Based on the results mentioned above, it should be emphasized here that the ambient temperature would not be an issue affecting the generation of ULH alignment by the hybrid voltage pulse. It has been proven that the strength of temperature elevation by dielectric heating can be predominated by 
voltage conditions [29], as shown in Figure 2a for example. Therefore, no matter how the ambient temperature is, the cell temperature can be increased to an appropriate value (e.g., a temperature higher than and near the clearing point $T_{\mathrm{c}}$ of the CLC material for inducing ULH alignment via phase and textural transitions, respectively) by optimizing the frequency and the amplitude of the leading component of the hybrid voltage pulse (i.e., the pulse at $f_{1}$ ). Moreover, according to the results of Figures 4 and 5 , when the frequency of the voltage pulse is switched from $f_{1}$ to $f_{2}$, the ULH alignment will be formed within the temperature range near but below $T_{\mathrm{c}}$, which is independent of the ambient temperature. The total elapsed time to form ULH alignment by the proposed method can be cut down at the expense of increasing the magnitude $V$ at $f_{1}$ (to shorten the heating time). However, such a waveform design in the operation pulse may be considered complicated in that $V$ at $f_{2}$ needs to be low enough to permit the induction of phase or textural transition.

To explain that the ULH, formed by the hybrid pulse of $V=30 \mathrm{~V}_{\mathrm{rms}}$ at $f_{1}=55 \mathrm{kHz}$ and $f_{2}=30 \mathrm{~Hz}$, is enabled and governed by EHDI, Figure $6 \mathrm{a}, \mathrm{b}$ show the real $\left(\varepsilon^{\prime}\right)$ - and imaginary $\left(\varepsilon^{\prime \prime}\right)$-part dielectric and the loss-tangent $(\tan \delta)$ spectra of the CLC cell in the P state at $T=25^{\circ} \mathrm{C}$ and $50{ }^{\circ} \mathrm{C}$, respectively. The probe voltage for dielectric measurements is as low as $0.5 \mathrm{~V}_{\mathrm{rms}}$, which is unable to give rise to the Fréedericksz transition and, in turn, the molecular reorientation. Previously, we have established that the EHDI-induced ULH alignment is determined by the transport of mobile ions and, thus, the strength of space charge polarization unraveled in the complex dielectric spectra. Using dielectric spectroscopy, an optimized frequency range between $f_{\mathrm{L}}$ and $f_{\mathrm{R}}$ to facilitate EHDI-induced ULH has also been identified [18]. It is worth to mention that $f_{\mathrm{L}}$ in Figure 6 is the lower frequency at which the $\varepsilon^{\prime}$ and $\varepsilon^{\prime \prime}$ curves intersect each other and the relaxation frequency $f_{\mathrm{R}}\left(>f_{\mathrm{L}}\right)$ refers to the one corresponding to the maximum value of the loss-tangent. As shown in Figure 6a, the value of $\varepsilon^{\prime}$ in the measured frequency regime $(20 \mathrm{~Hz}-100 \mathrm{kHz})$, ascribed to the molecular orientation, is nearly constant. Moreover, based on this role, it is reasonable for the absence of ULH alignment in the cell applied with $30 \mathrm{~Hz}$ voltages at $T=25^{\circ} \mathrm{C}$ in Figure 1a because both $f_{\mathrm{L}}$ and $f_{\mathrm{R}}$ fall lower than $20 \mathrm{~Hz}$, the lower frequency limit of the LCR meter E4980A. In contrast, as the temperature increases to $50{ }^{\circ} \mathrm{C}$, the three curves shift to higher frequencies due to the promoted ionic effect in the cell. As a consequence, $f_{\mathrm{L}}$ and $f_{\mathrm{R}}$ become $20 \mathrm{~Hz}$ and $63.2 \mathrm{~Hz}$ at $50{ }^{\circ} \mathrm{C}$, respectively, so that the EHDI-induced ULH alignment can be acquired by the pulse of $V=30 \mathrm{~V}_{\mathrm{rms}}$ at $f_{2}=30 \mathrm{~Hz}$ (note: $f_{\mathrm{L}}<f_{2}<f_{\mathrm{R}}$ ). This implies that when a CLC with low ion density is used, the ULH can be alternatively realized via the elevation in temperature and thus the resulting ionic effect by adjusting the frequency conditions of the hybrid voltage pulse to onset the EHDI.
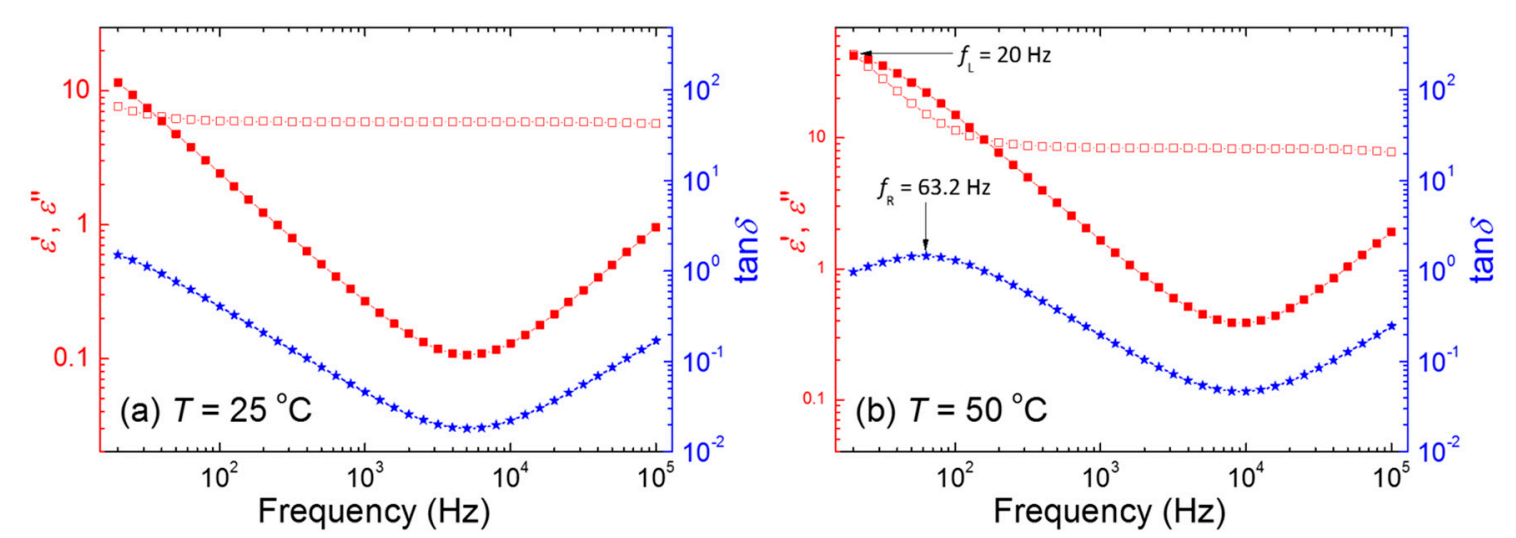

Figure 6. Complex dielectric and loss-tangent $(\tan \delta)$ spectra of the CLC cell in the P state at temperatures of (a) $\mathrm{T}=25^{\circ} \mathrm{C}$ and (b) $\mathrm{T}=50^{\circ} \mathrm{C}$.

\section{Conclusions}

Conventional methods to obtain a well-aligned ULH texture in a CLC cell with a simple surface-alignment condition (e.g., planar or hybrid) rely on the assisting voltage applied during 
the isotropic-to-CLC phase transition or via the EHDI. While the former requires a heating instrument in conjunction with a temperature controller to enable the phase transition so that the ULH can hardly switch to other CLC textures, the latter has been demonstrated specific for ion-rich positive CLCs. In the present work, we have referred to these two approaches and proposed to create ULH by applying a hybrid voltage across a CLC cell. The CLC used was injected into a $90^{\circ}$-twisted planar-aligned cell with nonzero electrode resistivity to enable dielectric heating via the pseudo-dielectric relaxation from the cell geometry [29].

With a hybrid voltage, the dielectric heating mechanism, which is dominated by the pseudo-dielectric relaxation originating from the ITO effect, permits the textural switching and phase transition in a CLC cell. On this basis under optimized voltage conditions, we have verified, by means of textural observations and measurements of time-dependent transmission and temperature, that the ULH alignment can be electrically generated through the thermal induction of the isotropic-to-CLC phase transition or by electrohydrodynamic instability in the homeotropic-to-CLC textural transition at a higher temperature to ensure a more pronounced ionic effect. The ULH obtained is switchable to another CLC texture, say, the Grandjean planar or focal conic state, by using the general scheme for the CLC textural switching. In contrast to the existing methods for the ULH formation reported in the literature, the proposed one presented in this study can adopt any CLC cell with simple surface alignment conditions (e.g., planar, $90^{\circ}$-twisted, and hybrid) and it is more universally applicable because ion-rich LCs and additional equipment for temperature controlling or mechanical shearing are not necessitated. Such an alternative approach holds great promise for overcoming the general hurdle of good-quality ULH, thereby facilitating its practical application for electro-optical devices.

Author Contributions: C.-H.Y. performed the experiments and drafted the manuscript. P.-C.W. conceived the experiments and helped analyze the data. Wei Lee supervised the whole study and finalized the manuscript.

Funding: This work was financially supported by the Ministry of Science and Technology, Taiwan, under grant Nos. 106-2923-M-009-002-MY3 and 107-2112-M-009-012-MY3.

Conflicts of Interest: The authors declare no conflict of interest.

\section{References}

1. Chilaya, G. Cholesteric Liquid Crystals: Optics, Electro-optics, and Photo-optics. In Chirality in Liquid Crystals; Kitzerow, H., Bahr, C., Eds.; Springer: New York, NY, USA, 2001; Chapter 6; pp. 159-185.

2. Huang, J.-C.; Hsiao, Y.-C.; Lin, Y.-T.; Lee, C.-R.; Lee, W. Electrically switchable organo-inorganic hybrid for a white-light laser source. Sci. Rep. 2016, 6, 28363. [CrossRef] [PubMed]

3. Hsiao, Y.-C.; Tang, C.-Y.; Lee, W. Fast-switching bistable cholesteric intensity modulator. Opt. Express 2011, 19, 9744-9749. [CrossRef]

4. White, T.J.; McConney, M.E.; Bunning, T.J. Dynamic color in stimuli-responsive cholesteric liquid crystals. J. Mater. Chem. 2016, 20, 9832-9847. [CrossRef]

5. Rudquist, P.; Komitov, L.; Lagerwall, S.T. Linear electro-optic effect in a cholesteric liquid crystal. Phys. Rev. E 1994, 50, 4735-4743. [CrossRef]

6. Musgrave, B.; Lehmann, P.; Coles, H.J. A new series of chiral nematic bimesogens for the flexoelectro-optic effect. Liq. Cryst. 1999, 26, 1235-1249. [CrossRef]

7. Coles, H.J.; Clarke, M.J.; Morris, S.M.; Broughton, B.J.; Blatch, A.E. Strong flexoelectric behavior in bimesogenic liquid crystals. J. Appl. Phys. 2006, 99, 034104. [CrossRef]

8. Morris, S.M.; Clarke, M.J.; Blatch, A.E.; Coles, H.J. Structure-flexoelastic properties of bimesogenic liquid crystals. Phys. Rev. E 2007, 75, 041701. [CrossRef]

9. Outram, B.I.; Elston, S.J. Frequency-dependent dielectric contribution of flexoelectricity allowing control of state switching in helicoidal liquid crystals. Phys. Rev. E 2013, 88, 012506. [CrossRef] [PubMed]

10. Varanytsia, A.; Chien, L.-C. Bimesogen-enhanced flexoelectro-optic behavior of polymer stabilized cholesteric liquid crystal. J. Appl. Phys. 2016, 11, 014502. [CrossRef]

11. Tan, G.; Lee, Y.-H.; Gou, F.; Hu, M.; Lan, Y.-F.; Tsai, C.-Y.; Wu, S.-T. Macroscopic model for analyzing the electro-optics of uniform lying helix cholesteric liquid crystals. J. Appl. Phys 2017, 121, 173102. [CrossRef] 
12. Patel, J.S.; Meyer, R.B. Flexoelectric electro-optics of a cholesteric liquid crystal. Phys. Rev. Lett. 1987, 58, 1538-1540. [CrossRef] [PubMed]

13. Salter, P.S.; Elston, S.J.; Raynes, P.; Parry-Jones, L.A. Alignment of the uniform lying helix structure in cholesteric liquid crystals. Jpn. J. Appl. Phys. 2009, 48, 101302. [CrossRef]

14. Rudquist, P.; Komitov, L.; Lagerwall, S.T. Volume-stabilized ULH structure for the flexoelectro-optic effect and the phase-shift effect in cholesterics. Liq. Cryst. 1998, 24, 329-334. [CrossRef]

15. Inoue, Y.; Moritake, H. Discovery of a transiently separable high-speed response component in cholesteric liquid crystals with a uniform lying helix. Appl. Phys. Express 2015, 8, 061701. [CrossRef]

16. Inoue, Y.; Moritake, H. Formation of a defect-free uniform lying helix in a thick cholesteric liquid crystal cell. Appl. Phys. Express 2015, 8, 071701. [CrossRef]

17. Wang, C.-T.; Wang, W.-Y.; Lin, T.-H. A stable and switchable uniform lying helix structure in cholesteric liquid crystals. Appl. Phys. Lett. 2011, 99, 041108. [CrossRef]

18. Nian, Y.-L.; Wu, P.-C.; Lee, W. Optimized frequency regime for the electrohydrodynamic induction of a uniformly lying helix structure. Photonics Res. 2016, 4, 227-232. [CrossRef]

19. Yu, C.-H.; Wu, P.-C.; Lee, W. Alternative generation of well-aligned uniform lying helix texture in a cholesteric liquid crystal cell. AIP Adv. 2017, 7, 105107. [CrossRef]

20. Park, K.-S.; Baek, J.-H.; Lee, Y.-J.; Kim, J.-H.; Yu, C.-J. Effects of pretilt angle and anchoring energy on alignment of uniformly lying helix mode. Liq. Cry. 2016, 43, 1184-1189. [CrossRef]

21. Gardiner, D.J.; Morris, S.M.; Hands, P.J.W.; Castles, F.; Qasim, M.M.; Kim, W.-S.; Choi, S.S.; Wikinson, T.D.; Coles, H.J. Spontaneous induction of the uniform lying helix alignment in bimesogenic liquid crystals for the flexoelectro-optic effect. Appl. Phys. Lett. 2012, 100, 063501. [CrossRef]

22. Li, C.-C.; Tseng, H.-Y.; Chen, C.-W.; Wang, C.-T.; Jau, H.-C.; Wu, Y.-C.; Hsu, W.-H.; Lin, T.-H. Tri-stable cholesteric liquid crystal smart window. SID DIGEST 2018, 49, 543-545. [CrossRef]

23. Hegde, G.; Komitov, L. Periodic anchoring condition for alignment of a short pitch cholesteric liquid crystal in uniform lying helix texture. Appl. Phys. Lett. 2010, 96, 113503. [CrossRef]

24. Outram, B.I.; Elston, S.J. Spontaneous and stable uniform lying helix liquid-crystal alignment. J. Appl. Phys. 2013, 113, 043103. [CrossRef]

25. Komitov, L.; Brown, G.P.B.; Wood, E.L.; Smout, A.B.J. Alignment of cholesteric liquid crystals using periodic anchoring. J. Appl. Phys. 1999, 86, 3508-3511. [CrossRef]

26. Carbone, G.; Salter, P.; Elston, S.J.; Raynes, P.; De Sio, L.; Ferjani, S.; Strangi, G.; Umeton, C.; Bartolino, R. Short pitch cholesteric electro-optical device based on periodic polymer structures. Appl. Phys. Lett. 2009, 9, 011102. [CrossRef]

27. Outram, B.I.; Elston, S.J.; Tuffin, R.; Siemianowski, S.; Snow, B. The use of mould-templated surface structures for high-quality uniform-lying-helix liquid-crystal alignment. J. Appl. Phys. 2013, 113, 213111. [CrossRef]

28. Carbone, G.; Corbett, D.; Elston, S.J.; Raynes, P.; Jesacher, A.; Simmonds, R.; Booth, M. Uniform lying helix alignment on periodic surface relief structure generated via laser scanning lithography. Mol. Cryst. Liq. Cryst. 2011, 544, 37-49. [CrossRef]

29. Wu, P.-C.; Wu, G.-W.; Timofeev, I.V.; Zyryanov, V. Ya.; Lee, W. Electro-thermally tunable reflective colors in a self-organized cholesteric helical superstructure. Photonics Res. 2018, 6, 1094-1100. [CrossRef]

30. Wu, P.-C.; Hsiao, C.-Y.; Lee, W. Photonic bandgap-cholesteric device with electrical tunability and optical tristability in its defect modes. Crystals 2017, 7, 184. [CrossRef]

31. Schadt, M. Dielectric heating and relaxations in nematic liquid crystals. Mol. Cryst. Liq. Cryst. 1981, 66, 319-336. [CrossRef]

32. Jian, B.-R.; Tang, C.-Y.; Lee, W. Temperature-dependent electrical properties of dilute suspensions of carbon nanotubes in nematic liquid crystals. Carbon 2011, 49, 910-914. [CrossRef]

(C) 2019 by the authors. Licensee MDPI, Basel, Switzerland. This article is an open access article distributed under the terms and conditions of the Creative Commons Attribution (CC BY) license (http://creativecommons.org/licenses/by/4.0/). 